\title{
Chest radiography is a poor predictor of respiratory symptoms and functional impairment in survivors of severe COVID-19 pneumonia
}

\author{
Rebecca F. D'Cruz (10) ${ }^{1}$, Michael D. Waller (10) ${ }^{1,2}$, Felicity Perrin ${ }^{2}$, \\ Jimstan Periselneris (10) ${ }^{2}$, Sam Norton (103 ${ }^{3}$, Laura-Jane Smith (102 ${ }^{2}$, Tanya Patrick ${ }^{2}$, \\ David Walder (i) $^{2}$, Amadea Heitmann ${ }^{2}$, Kai Lee ${ }^{2}$, Rajiv Madula², \\ William McNulty², Patricia Macedo (1) ${ }^{2}$, Rebecca Lyall², Geoffrey Warwick², \\ James B. Galloway $\mathbb{1}^{3}$, Surinder S. Birring $\mathbb{1}^{1,2}$, Amit Patel $^{2}$, Irem Patel ${ }^{1,2}$ and \\ Caroline J. Jolley (ib ${ }^{1,2}$
}

\section{ABSTRACT}

Background: A standardised approach to assessing COVID-19 survivors has not been established, largely due to the paucity of data on medium- and long-term sequelae. Interval chest radiography is recommended following community-acquired pneumonia; however, its utility in monitoring recovery from COVID-19 pneumonia remains unclear.

Methods: This was a prospective single-centre observational cohort study. Patients hospitalised with severe COVID-19 pneumonia (admission duration $\geqslant 48 \mathrm{~h}$ and oxygen requirement $\geqslant 40 \%$ or critical care admission) underwent face-to-face assessment at 4-6 weeks post-discharge. The primary outcome was radiological resolution of COVID-19 pneumonitis (Radiographic Assessment of Lung Oedema score <5). Secondary outcomes included clinical outcomes, symptom questionnaires, mental health screening (Trauma Screening Questionnaire, seven-item Generalised Anxiety Disorder assessment and nine-item Patient Health Questionnaire) and physiological testing (4-m gait speed (4MGS) and 1-min Sit-to-Stand (STS) tests).

Results: 119 patients were assessed between June 3, 2020 and July 2, 2020 at median (interquartile range (IQR)) 61 (51-67) days post-discharge: mean \pm SD age $58.7 \pm 14.4$ years, median (IQR) body mass index 30.0 $(25.9-35.2) \mathrm{kg} \cdot \mathrm{m}^{-2}, 62 \%$ male and $70 \%$ ethnic minority. Despite radiographic resolution of pulmonary infiltrates in $87 \%$, modified Medical Research Council Dyspnoea (breathlessness) scale grades were above pre-COVID-19 baseline in 44\%, and patients reported persistent fatigue (68\%), sleep disturbance (57\%) and breathlessness (32\%). Screening thresholds were breached for post-traumatic stress disorder (25\%), anxiety $(22 \%)$ and depression (18\%). 4MGS was slow $\left(<0.8 \mathrm{~m} \cdot \mathrm{s}^{-1}\right)$ in $38 \%$ and $35 \%$ desaturated by $\geqslant 4 \%$ during the STS test. Of 56 thoracic computed tomography scans performed, 75\% demonstrated COVID-19-related interstitial and/or airways disease.

Conclusions: Persistent symptoms, adverse mental health outcomes and physiological impairment are common 2 months after severe COVID-19 pneumonia. Follow-up chest radiography is a poor marker of recovery; therefore, holistic face-to-face assessment is recommended to facilitate early recognition and management of post-COVID-19 sequelae.

@ERSpublications

At 2-month follow-up, survivors of severe \#COVID19 pneumonia experience persistent symptoms, functional disability and mental health problems despite radiographic resolution occurring in the majority, highlighting the need for holistic follow-up care https://bit.ly/3dz $8 \mathrm{dXb}$

Cite this article as: D'Cruz RF, Waller MD, Perrin F, et al. Chest radiography is a poor predictor of respiratory symptoms and functional impairment in survivors of severe COVID-19 pneumonia. ERJ Open Res 2021; 7: 00655-2020 [https://doi.org/10.1183/23120541.00655-2020].

This article has supplementary material available from openres.ersjournals.com

Received: 9 Sept 2020 | Accepted: 7 Oct 2020

Copyright $\odot$ ERS 2021. This article is open access and distributed under the terms of the Creative Commons Attribution Non-Commercial Licence 4.0. 


\section{Introduction}

Following alarmingly rapid global spread of the novel severe acute respiratory syndrome coronavirus 2 (SARS-CoV-2), COVID-19 was categorised as a pandemic on March 11, 2020 [1]. Acute manifestations of the disease are widely reported, with fever, cough and breathlessness recognised as the most common presenting symptoms [2]. Following the acute phase of illness there is increasing anecdotal awareness of patients with "long COVID" in whom residual symptoms persist beyond the acute viral illness [3]. However, a robust evidence base on medium- and long-term physical and psychological sequelae of severe COVID-19 infection is currently lacking.

Drawing on experience from previous coronavirus global outbreaks (SARS-CoV-1 (SARS) in 2002-2004 and Middle East respiratory syndrome-related coronavirus (MERS) in 2012) and our comprehensive understanding of outcomes following acute respiratory distress syndrome (ARDS) and critical illness, COVID-19 survivors are anticipated to be at risk of impaired lung function [4], interstitial lung disease [5], exercise limitation and impaired quality of life in the months and years following hospital discharge $[4,6,7]$. Given the $14 \%$ prevalence of post-traumatic stress disorder (PTSD) in critical illness survivors [8] and excess increase in mental distress observed among UK adults during the COVID-19 pandemic [9], the burden of mental health disorders following COVID-19 infection is expected to be high. With 19.6 million cases now (August 2020) confirmed globally and the daily incidence continuing to climb [10], it is apparent that early recognition and management of complications among the increasing population of severe COVID-19 survivors, many of whom will have experienced multiorgan involvement, critical care admission and psychological trauma, is a clinical priority.

We aimed to prospectively investigate clinical, radiological, functional and psychological COVID-19 sequalae of severe COVID-19 pneumonia, and to identify factors associated with symptomatic and functional recovery.

\section{Methods}

\section{Study design and participants}

This single-centre prospective observational cohort study was conducted at King's College Hospital, an urban university hospital in London (UK). We analysed routine data of COVID-19 survivors attending the King's College Hospital COVID-19 post-discharge clinical service. To identify patients hospitalised with COVID-19 pneumonia, we screened electronic medical records of consecutive patients aged $\geqslant 18$ years hospitalised with PCR-confirmed COVID-19 on naso- and oropharyngeal swab between February and May 2020. In the UK healthcare system during the pandemic, patients presenting to the emergency department with symptoms consistent with COVID-19 were admitted to hospital if they were considered at higher risk of severe disease (based on age, comorbidities or social circumstances) and/or they had significantly abnormal vital observations or investigations (venous or arterial blood results and chest radiography). We defined severe COVID-19 pneumonia as requiring hospitalisation for $\geqslant 48 \mathrm{~h}$ and inspiratory oxygen fraction $\left(F_{\mathrm{IO}_{2}}\right) \geqslant 40 \%$ or intensive care unit (ICU) admission. Patients fulfilling these criteria and surviving to discharge were invited to attend the clinic for face-to-face assessment in accordance with British Thoracic Society guidance [11]. Herein, we report the first month of prospectively collected data from consecutive patients assessed between June 3, 2020 and July 2, 2020.

This study was approved by the Clinical Governance committee, King's College NHS Foundation Trust, judged to be a service evaluation exempt from NHS Research Ethics Committee review, since no a priori hypothesis testing, randomisation or treatment allocation was undertaken, and conducted according to the principles of the Declaration of Helsinki [12].

\section{Data collection}

Demographic and anthropometric data and inpatient clinical outcomes for all patients screened were obtained from medical records. A summary of follow-up data collected is provided in supplementary table S1. Questionnaires were used to evaluate persistent symptoms, self-reported functional disability, depression, anxiety, PTSD and cognition [13-20]. Functional disability was objectively assessed using the 4-m gait speed (4MGS) and 1-min Sit-to-Stand (STS) tests [21, 22]. Admission, worst inpatient and follow-up radiographs were graded using the Radiographic Assessment of Lung Oedema (RALE) score [23].

Affiliations: ${ }^{1}$ Centre for Human and Applied Physiological Sciences, King's College London, London, UK. ${ }^{2}$ Dept of Respiratory Medicine, King's College Hospital NHS Foundation Trust, London, UK. ${ }^{3}$ Centre for Rheumatic Disease, King's College London, London, UK.

Correspondence: Caroline J. Jolley, Centre for Human and Applied Physiological Sciences, Faculty of Life Sciences and Medicine, King's College London, Denmark Hill, London, SE5 9RS, UK.

E-mail: caroline.jolley@akcl.ac.uk 
Thoracic computed tomography (CT) scans were performed for patients with persistent chest radiograph abnormalities, respiratory symptoms or oxygen desaturation $\geqslant 4 \%$ during the STS test. Additional methodological details are provided in the supplementary material.

\section{Outcomes}

The primary outcome of this study was radiological resolution of COVID-19 pneumonia, in accordance with national guidelines [11]. Secondary outcomes included demographics, anthropometrics, inpatient clinical course, symptom questionnaires, mental health screening and physiological testing (STS repetitions and oxygen desaturation, and $4 \mathrm{MGS}$ ).

\section{Statistical analysis}

The aim of this study was to perform rapid characterisation of severe COVID-19 recovery, with no a priori hypothesis testing or sample size calculation. Consecutive survivors of confirmed severe COVID-19 pneumonia attending face-to-face assessments were included. Data are presented as mean with standard deviation or median (interquartile range (IQR)) for continuous variables, depending on the normality of the data, and frequency (percentage $(95 \%$ confidence interval)) for categorical variables. Group comparisons were performed using independent t-tests and Chi-squared tests. Ordinal logistic regression modelling was used to identify factors associated with measures of COVID-19 recovery. Odds ratios (adjusted for age, sex and ethnicity) are presented. Statistical significance was concluded at the two-sided significance level of 0.05 . Analyses were conducted with SPSS version 26 (IBM, Armonk, NY, USA).

\section{Results}

\section{Baseline and inpatient characteristics}

Between June 3, 2020 and July 2, 2020, 143 patients were invited to attend face-to-face assessment post-discharge, of whom 119 attended. These patients had been hospitalised between March 5, 2020 and May 28, 2020, during which time a total of 898 patients were hospitalised with confirmed COVID-19, of whom 657 survived to discharge (figure 1). Data for all patients hospitalised with COVID-19, those surviving to discharge and those that did not attend their post-COVID-19 clinic appointment, and between-group comparisons are provided in supplementary tables S2 and S3. Baseline demographic and anthropometric characteristics at follow-up and patients' inpatient clinical course are presented in table 1. Mean \pm SD age was $58.7 \pm 14.4$ years, median (IQR) body mass index (BMI) was $30.0(25.9-35.2) \mathrm{kg} \cdot \mathrm{m}^{-2}$, $62 \%$ of patients were male and $70 \%$ self-reported as Black, Asian or minority ethnic. Median (IQR) Charlson Comorbidity Index was 2 (1-4), 53\% had pre-existing cardiovascular disease, $11 \%$ had obstructive lung disease, $7 \%$ had end-stage renal failure, $18 \%$ had no pre-existing comorbidities. Patients were moderately hypoxaemic at admission (median (IQR) arterial oxygen tension $\left(P_{\mathrm{aO}_{2}}\right) / F_{\mathrm{IO}_{2}} \quad 169$ (106-272)). 58\% of patients were lymphopenic and 20\% were thrombocytopenic. 41 (34\%) were admitted to the ICU, 34 (29\%) received invasive mechanical ventilation (IMV) and median (IQR) ICU admission duration was 14.5 (7-27) days. Mean \pm SD hospital length of stay for critical care patients was $30.8 \pm 16.3$ days and median (IQR) 9 (7-14.5) days for those receiving ward-based care. 70 (59\%) experienced at least one complication attributable to COVID-19 (supplementary table S4), of which acute kidney injury (35\%) and venous thromboembolism $(23 \%)$ were the most common.

\section{Follow-up characteristics}

Median (IQR) times between hospital admission and discharge and follow-up assessment were 76 (71-83) and 61 (51-67) days, respectively. Time between discharge and clinic assessment and current modified Medical Research Council (mMRC) Dyspnoea (breathlessness) scale grade is displayed in figure 2. 57 (48\%) patients utilised hospital services following hospital discharge: 23 (40\%) attended outpatient appointments for monitoring of inpatient complications (haematology, renal, diabetes), 16 (28\%) attended the emergency department, nine (16\%) were re-hospitalised and nine (16\%) attended planned outpatient appointments for pre-existing comorbidities.

Questionnaire scores are displayed in figure 3. Median (IQR) mMRC grade at follow-up was 1 (0-2) and pre-COVID-19 was $0(0-1), 51$ out of 115 (44.3.2\%, 95\% CI 35.8-53.0\%) patients had not returned to pre-COVID-19 mMRC baseline. The association between current mMRC grade and time between discharge and clinical assessment was weak $\left(\mathrm{R}^{2}<0.001, \mathrm{p}=0.82\right)$. Post-COVID-19 Functional Status (PCFS) grade was $\geqslant 2$ in 47 out of $115(40.9 \%, 95 \%$ CI $33.0-47.8 \%)$. Of those whose mMRC grade had not returned to pre-COVID-19 baseline, 10 out of 51 (19.6\%, 95\% CI 8.6-31.9\%) had no pre-existing comorbidity. Comorbid obstructive lung disease was associated with failure of mMRC recovery to baseline (OR 5.06, 95\% CI 1.33-19.24; $\mathrm{p}=0.017$ ) and PCFS grade $\geqslant 2$ (OR 2.84, 95\% CI 1.01-7.98; p=0.047) (table 2).

Median (IQR) number of persistent symptoms was 4 (2-5), 11\% of patients reported no persistent symptoms. Burdensome breathlessness (numerical rating scale (NRS) breathlessness $\geqslant 4$ ) was present in 37 


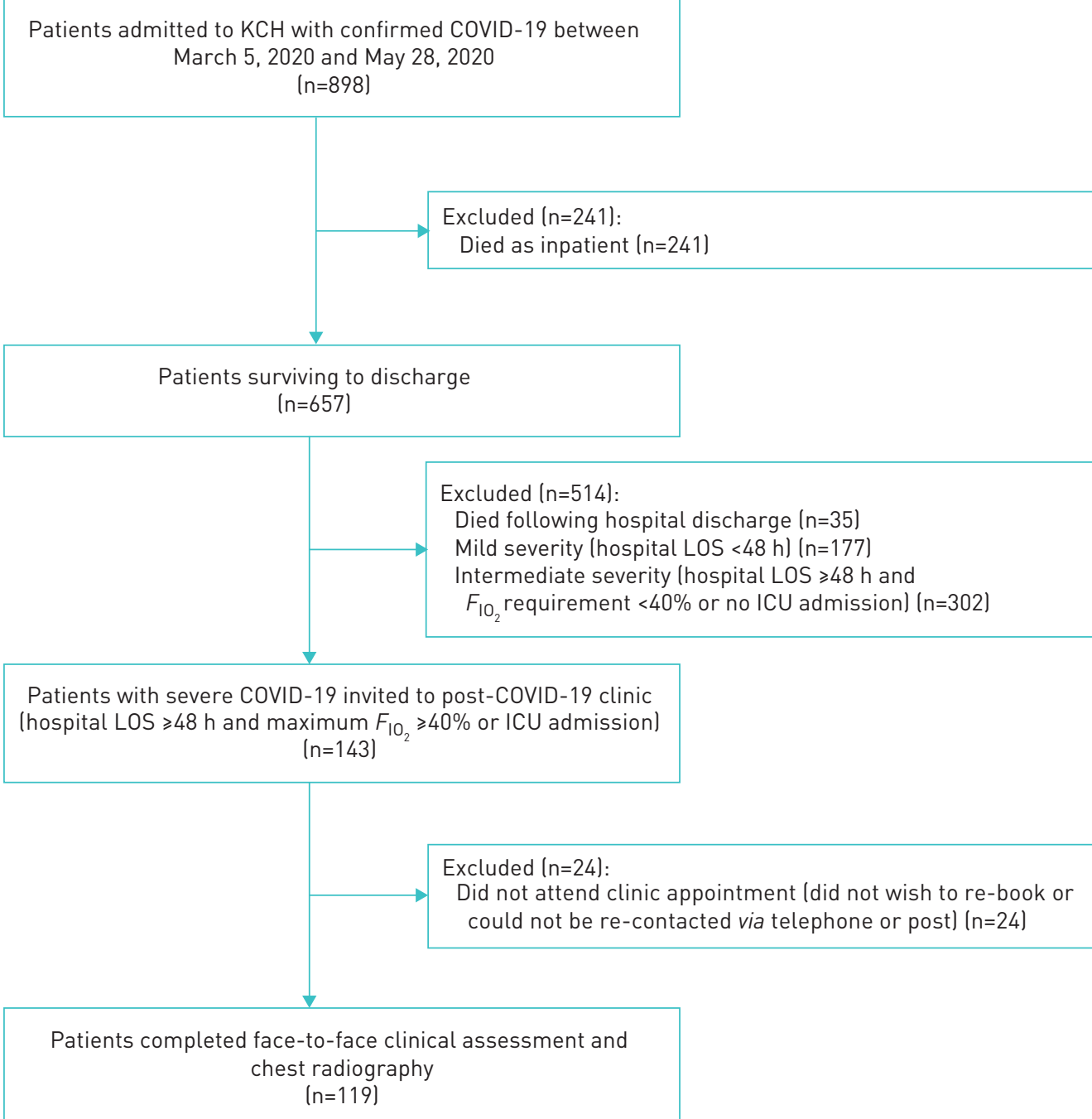

FIGURE 1 Patient flowchart. KCH: King's College Hospital; LOS: length of stay; $F_{\mathrm{IO}_{2}}$ : inspiratory oxygen fraction; ICU: intensive care unit.

out of $115(32.2 \%, 95 \%$ CI $25.2-40.0 \%)$. Persistent cough (NRS $\geqslant 1)$ was present in 49 out of $115(42.6 \%$, 95\% CI 33.9-52.2\%) and burdensome cough (NRS $\geqslant 4$ ) in eight out of 115 (6.9\%, 95\% CI 3.5-10.4\%). 78 out of 115 (67.8\%, 95\% CI 60.0-74.8\%) reported fatigue, 65 out of 115 (56.5\%, 95\% CI 47.3-66.1\%) reported sleep disturbance and 57 out of 115 (49.6\%, 95\% CI 40.9-58.3\%) reported pain. Where stated, pain was most commonly reported in the shoulder $(n=9(29 \%))$, chest $(n=7(23 \%))$, lower limbs $(n=6$ $(19 \%))$ and back $(n=4(13 \%))$. Pre-morbid obstructive lung disease was associated with persistent $(\mathrm{NRS} \geqslant 1)$ breathlessness (OR 8.04, 95\% CI 0.19-21.4; $\mathrm{p}=0.03$ ) and cough (OR 3.43, 95\% CI 0.98-12.0), but not burdensome (NRS $\geqslant 4$ ) breathlessness or cough (OR 1.97, 95\% CI 0.60-6.47; p=0.26 and OR 2.27, 95\% CI $0.38-13.69 ; \mathrm{p}=0.37$, respectively). There were no associations between the presence or absence of pre-existing comorbidities and persistent fatigue, sleep disturbance or pain. The nine-item Patient Health Questionnaire (PHQ-9) (depression) score was $>9$ in 20 out of 111 (18.0\%, 95\% CI 11.7-23.4\%) and the seven-item Generalised Anxiety Disorder (GAD-7) assessment score was >9 in 25 out of $113(22.1 \%, 95 \%$ CI $15.0-29.8 \%) .28$ out of $113(24.8 \%, 95 \%$ CI $18.1-31.9 \%)$ scored $\geqslant 6$ on the Trauma Screen Questionnaire. 21 out of $97(21.6 \%, 95 \%$ CI $14.4-28.9 \%)$ scored $\geqslant 8$ on the six-item Cognitive Impairment Test.

Physiological outcomes are displayed in table 3. Resting arterial oxygen saturation measured by pulse oximetry $\left(S_{\mathrm{pO}_{2}}\right)$ was $<94 \%$ in two out of $119(1.7 \%)$ patients. $115(97 \%)$ completed the 4MGS. Mean \pm SD 4MGS was $0.87 \pm 0.29 \mathrm{~m} \cdot \mathrm{s}^{-1}$ and $44(38 \%)$ had $4 \mathrm{MGS}<0.8 \mathrm{~m} \cdot \mathrm{s}^{-1} .109(92 \%)$ completed the STS. The number of repetitions performed was $<2.5$ th percentile in 56 (52\%). 39 (35\%) desaturated by $\geqslant 4 \%$ and 13 (12\%) desaturated to $S_{\mathrm{pO}_{2}} \leqslant 88 \%$. There were no adverse events during physiological testing. There were no associations between pre-morbid obstructive lung disease and physiological functional impairment (OR 0.68, 95\% CI 0.16-2.95; p=0.605) (table 2); however, cardiovascular disease was associated with 4MGS 


\section{TABLE 1 Baseline characteristics at follow-up and inpatient clinical course}

\begin{tabular}{|c|c|}
\hline Age years $(n=119)$ & $58.7 \pm 14.4$ \\
\hline $18-29$ & $4(3.4,0.8-6.7)$ \\
\hline $30-39$ & $11(9.2,5.0-14.3)$ \\
\hline $40-49$ & $13(10.9,6.7-15.1)$ \\
\hline $50-59$ & $36(30.3,22.7-38.7)$ \\
\hline $60-69$ & $27(22.7,16.0-28.6)$ \\
\hline $70-79$ & $18(15.1,10.1-21.0)$ \\
\hline$\geqslant 80$ & $10(8.4,5.0-12.6)$ \\
\hline \multicolumn{2}{|l|}{ Sex $(n=119)$} \\
\hline Female & $45(37.8,29.4-46.2)$ \\
\hline Male & $74(62.2,53.8-70.6)$ \\
\hline \multicolumn{2}{|l|}{ Ethnicity (n=119) } \\
\hline BAME (yes/no) & $83(69.7,61.3-78.2)$ \\
\hline White & $36(30.3,22.6-37.8)$ \\
\hline Black & $52(43.7,36.1-51.3)$ \\
\hline Asian & $18(15.1,10.1-20.2)$ \\
\hline Mixed race & $5(4.2,1.7-6.7)$ \\
\hline Other & $8(6.7,3.4-10.9)$ \\
\hline Index of multiple deprivation score ( $n=115$ ) & $26.6 \pm 9.7$ \\
\hline BMI $\mathrm{kg} \cdot \mathrm{m}^{-2}(\mathrm{n}=118)$ & $30.0(25.9-35.2)$ \\
\hline Underweight $(<18.5)$ & $0(0.0)$ \\
\hline Normal (18.5-24.9) & $22(18.6,12.7-24.6)$ \\
\hline Overweight (25.0-29.9) & $35(29.7,22.9-37.3)$ \\
\hline Obese (30.0-34.9) & $30(25.4,19.5-33.1)$ \\
\hline Severely obese (35.0-39.9) & $20(16.9,11.0-22.0)$ \\
\hline Morbidly obese (40.0-49.9) & $9(7.6,4.2-11.0)$ \\
\hline Super obese $(\geqslant 50.0)$ & $2(1.7,0.0-4.2)$ \\
\hline \multicolumn{2}{|l|}{ Smoking status $(n=110)$} \\
\hline Never-smoker & $82(74.5,67.3-82.7)$ \\
\hline Ex-smoker & $25(22.7,16.4-28.2)$ \\
\hline Current smoker & $3(2.7,0.0-6.4)$ \\
\hline \multicolumn{2}{|l|}{ Comorbidities ( $n=119)$} \\
\hline Charlson Comorbidity Index & $2(1-4)$ \\
\hline Any cardiovascular disease & $63(52.9,44.5-61.8)$ \\
\hline Hypertension & $54(45.4,37.7-52.9)$ \\
\hline Hyperlipidaemia & $25(21.0,15.1-27.4)$ \\
\hline Ischaemic heart disease/heart failure & $8(6.7,3.4-10.9)$ \\
\hline Diabetes & $41(34.5,26.4-42.9)$ \\
\hline Immunosuppressed & $16(13.4,8.4-18.5)$ \\
\hline Obstructive lung disease & $13(10.9,6.7-16.0)$ \\
\hline Malignancy & $12(10.1,5.9-14.3)$ \\
\hline End-stage renal failure & $8(6.7,3.4-10.1)$ \\
\hline Thyroid disease & $7(5.9,2.5-9.2)$ \\
\hline Mental health condition & $6(5.0,2.5-7.6)$ \\
\hline Cerebrovascular disease & $5(4.2,1.7-6.7)$ \\
\hline Admission $P_{\mathrm{aO}_{2}} / F_{\mathrm{IO}_{2}}$ & $168.8(105.9-272.3)$ \\
\hline \multicolumn{2}{|l|}{$P_{\mathrm{aO}_{2}} / F_{\mathrm{IO}_{2}}$ severity (n=119) } \\
\hline$>300$ (normal) & $13(14.6,9.0-21.3)$ \\
\hline 200-300 (mild) & $23(25.8,19.1-34.8)$ \\
\hline 100-199 (moderate) & $32(36.0,28.1-44.9)$ \\
\hline$<100$ (severe) & $21(23.6,15.7-32.6)$ \\
\hline \multicolumn{2}{|l|}{ Maximum respiratory support ( $n=119$ ) } \\
\hline $\mathrm{FMO}_{2}$ & $71(59.7,51.3-67.2)$ \\
\hline PAP & $14(11.8,6.9-16.8)$ \\
\hline IMV & $34(28.6,21.0-37.0)$ \\
\hline \multicolumn{2}{|l|}{ COVID-19 complications ( $n=119$ ) } \\
\hline None during admission & $49(41.2,33.6-48.7)$ \\
\hline Venous thromboembolism & $27(22.7,16.8-29.4)$ \\
\hline Pulmonary embolism & $23(19.3,12.6-26.1)$ \\
\hline Deep vein thrombosis & $6(5.0,2.5-7.6)$ \\
\hline
\end{tabular}




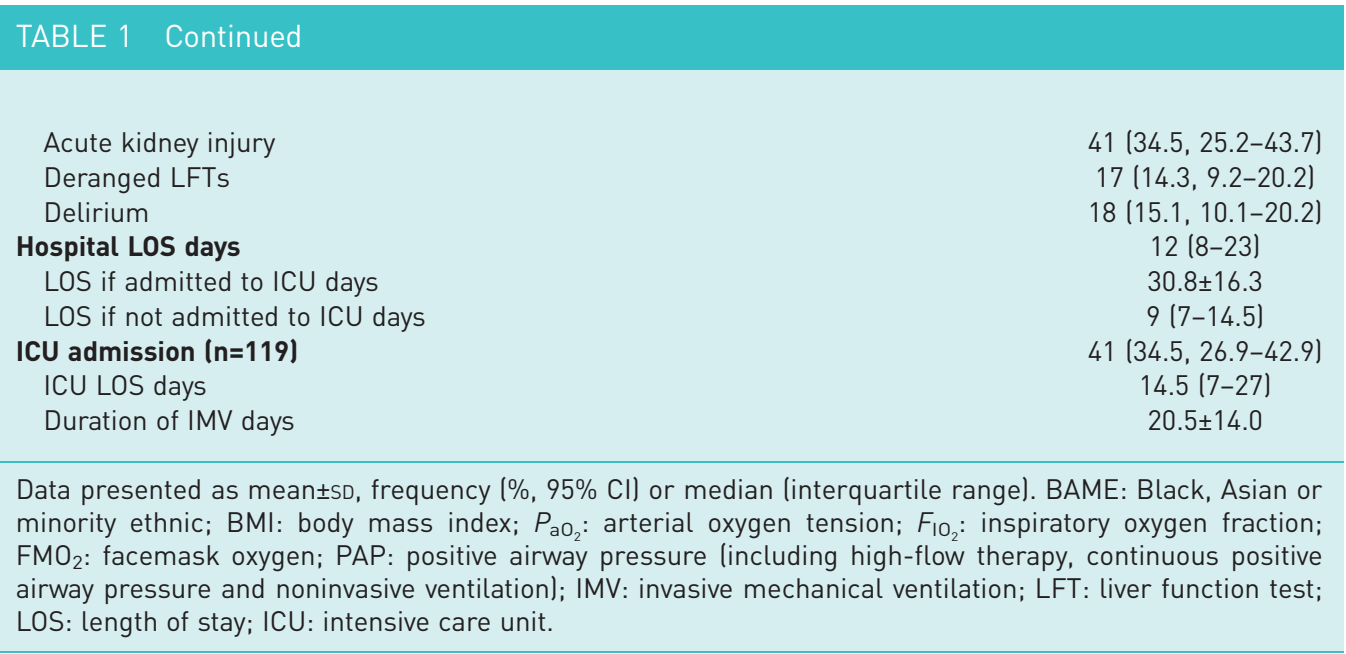

$<0.8 \mathrm{~m} \cdot \mathrm{s}^{-1}$ (OR 3.95, 95\% CI 0.42-2.49; $\mathrm{p}=0.003$ ). There were no associations between pre-existing comorbidities and exertional oxygen desaturation $(\geqslant 4 \%)$ during STS testing. There was no relationship between age categories (as defined in table 1) and persistent post-COVID-19 symptoms, self-reported functional disability (mMRC grade not returned to baseline or PCFS grade $\geqslant 2$ ) or physiological impairment (4MGS $<0.8 \mathrm{~m} \cdot \mathrm{s}^{-1}$ or oxygen desaturation $\geqslant 4 \%$ ).

All 119 patients underwent chest radiography at follow-up assessment. RALE scores at admission, peak of inpatient clinical illness and follow-up are presented in figure 4. Only 15 (13\%) had evidence of COVID-19-related lung disease on follow-up radiography (RALE score >4). 56 patients were invited for follow-up CT and pulmonary angiography based on abnormal chest radiography, persistent respiratory symptoms or exercise desaturation. Of these, 42 scans demonstrated features of COVID-19-related interstitial lung disease and/or airways disease: 21 (37.5\%, 95\% CI 26.8-48.2\%) revealed ground-glass/ organising pneumonia, nine (16.1\%, 95\% CI 8.9-25.0\%) revealed small airways disease/bronchiectasis, five (8.9\%, 95\% CI 3.6-16.1\%) revealed a combination of interstitial patterns, four $(7.1 \%, 95 \%$ CI $1.8-14.3 \%)$ had a combination of interstitial and airways changes, and three (5.4\%, 95\% CI $0.0-12.5 \%)$ revealed fibrosis/nonspecific interstitial pneumonia. 14 (26.2\%, 95\% CI 15.1-37.7\%) CT scans were normal or had no abnormality to explain persistent symptoms or desaturation. No pulmonary emboli were identified on CT pulmonary angiography. Presence of COVID-19-related CT abnormalities was associated with mental health screening questionnaires (PHQ-9 >9, GAD-7 >9 and/or Trauma Screening Questionnaire $\geqslant 6$ ) (Chi-squared $=3.98,95 \%$ CI $0.02-0.54 ; \mathrm{p}=0.046$ ), but not with any measure of patient-reported or physiological functional impairment (mMRC, PCFS, 4MGS $<0.8 \mathrm{~m} \cdot \mathrm{s}^{-1}$ or oxygen desaturation $\geqslant 4 \%$ during STS testing). Only $21 \%$ of patients with abnormal CT findings also had an abnormal follow-up chest radiograph; however, $78 \%$ of those with oxygen desaturation $\geqslant 4 \%$ during STS also had abnormal CT findings. 33 patients had a normal chest radiograph (RALE score 0-4) and an abnormal CT scan; nine patients had both an abnormal chest radiograph (RALE score $>4$ ) and an abnormal CT scan. Among those with abnormal CT scans, presence or absence of radiographic abnormalities was not predictive of any patient-reported or physiological outcome measure.

FIGURE 2 Relationship between modified Medical Research Council (mMRC) Dyspnoea (breathlessness) scale grade and time from hospital discharge. Black line: linear regression line (to indicate the line of best fit); grey lines: $95 \% \mathrm{Cl}$.

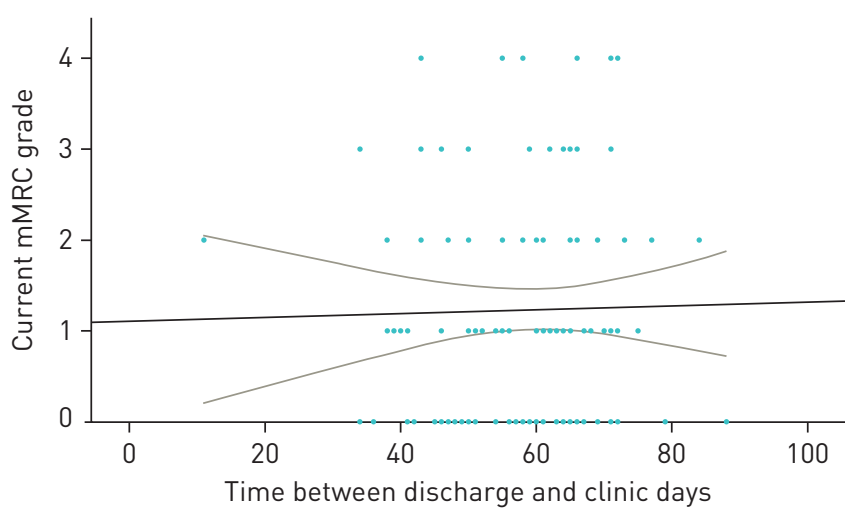



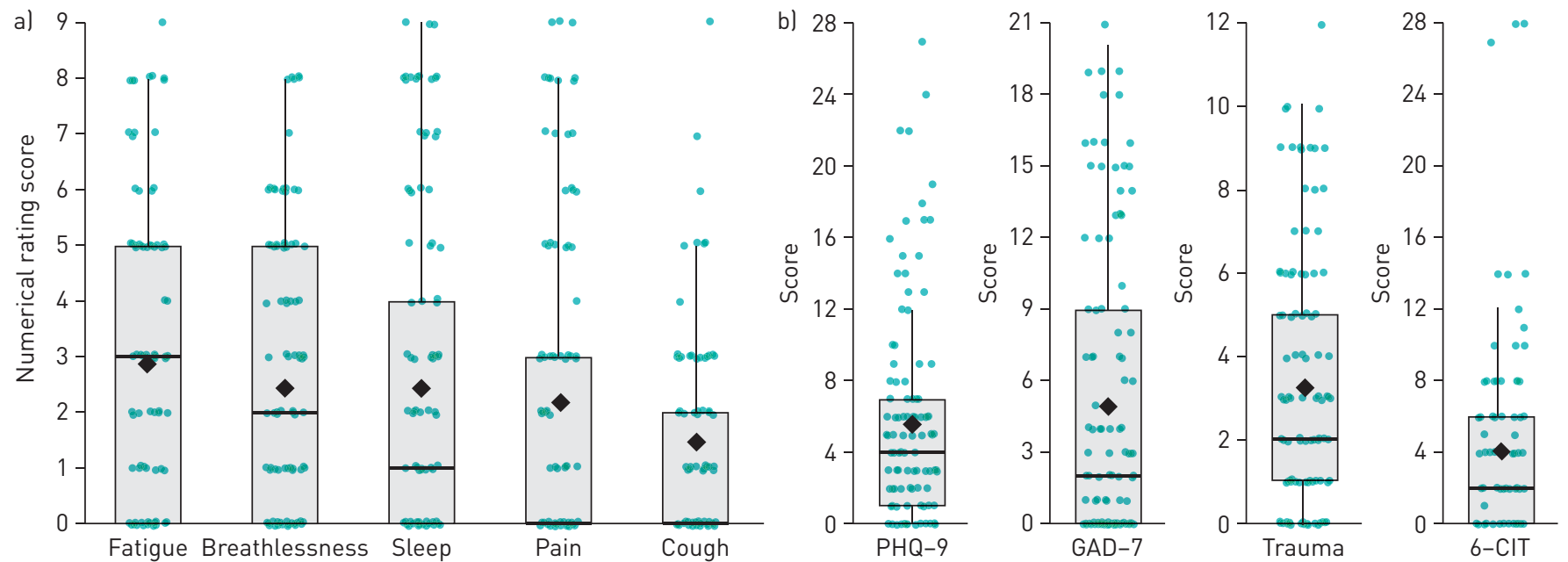

FIGURE 3 Box plots of persistent symptoms and mental health and neurocognitive outcomes. PHQ-9: nine-item Patient Health Questionnaire; GAD-7: seven-item Generalised Anxiety Disorder assessment; 6-CIT: six-item Cognitive Impairment Test. a) Numerical rating scores for fatigue, breathlessness, sleep, pain and cough. b) Scores for PHQ-9, GAD-7, Trauma Screening Questionnaire and 6-CIT. Observed values are indicated by circles. Shaded boxes indicate interquartile range with median indicated by the midline and mean indicated by a diamond. The lower/upper whiskers represent the range to the minimum/maximum value or 1.5 times the median to the lower/upper quartile, whichever is highest.

Ordinal logistic regression modelling was performed for the outcomes of return of mMRC grade to pre-COVID-19 baseline, PCFS grade $\geqslant 2$, positive mental health screening (PHQ-9 or GAD-7 $>9$ or Trauma Screening Questionnaire $\geqslant 6$ ) and physiological functional impairment (4MGS $<0.8 \mathrm{~m} \cdot \mathrm{s}^{-1}$, STS repetitions $<2.5$ th percentile or oxygen desaturation $\geqslant 4 \%$ on STS) (table 3 ). Positive associations were found between PCFS grade $\geqslant 2$, physiological impairment (4MGS $<0.8 \mathrm{~m} \cdot \mathrm{s}^{-1}$ and STS repetitions $<2.5$ th percentile) and positive mental health screening. Critical care admission and need for IMV were associated with physiological functional impairment. Neither worst inpatient nor follow-up RALE score were associated with any modelled outcome measure.

\section{Discussion \\ Principal findings}

This is the first study to provide holistic characterisation of medium-term sequelae 2 months following severe COVID-19 pneumonia incorporating clinical, radiological, physiological and psychological outcome measures. At 7-9 weeks following index hospitalisation, chest radiography was a poor marker of abnormal CT findings and persistent functional disability. $87 \%$ of patients had a RALE score $0-4.75 \%$ of CT scans demonstrated COVID-19-related interstitial lung disease and/or airways disease; however, only $21 \%$ of patients with abnormal CT findings also had an abnormal follow-up chest radiograph.

The burdens of persistent symptoms, mental health disorders and functional disability 2 months following index hospitalisation were high, with persistent fatigue (68\%) and sleep disturbance (57\%) more prevalent than respiratory symptoms (breathlessness $32 \%$ and cough $7 \%$ ). Significant depression and anxiety was present in $18 \%$ and $22 \%$ of patients, respectively, and $25 \%$ screened positive for PTSD. $41 \%$ of patients reported persistent limitations in everyday life due to COVID-19 (PCFS grade $\geqslant 2$ ) and mMRC grade failed to return to pre-COVID-19 baseline in 44\%. Face-to-face assessment was invaluable in identifying the high prevalence of objective functional impairment, evident during physiological testing: 4MGS was $<0.8 \mathrm{~m} \cdot \mathrm{s}^{-1}$ in $38 \%$, the number of repetitions was $<2.5$ th percentile for age and sex in $52 \%$ of patients performing the STS test, and $35 \%$ desaturated by $\geqslant 4 \%$ during the STS test.

\section{Comparison with other studies}

The baseline characteristics and inpatient clinical course of this cohort are highly consistent with national data for COVID-19 critical care admissions with regard to age, sex, BMI, $P_{\mathrm{aO}_{2}} / F_{\mathrm{IO}_{2}}$ severity, proportion requiring IMV and duration of ICU admission [24]. The higher proportion of patients from an ethnic minority background is consistent with the local population served by our hospital. Data from 20133 patients hospitalised with COVID-19 in the UK between February and April 2020 demonstrated a 26\% inpatient mortality, comparable to the $27 \%$ inpatient mortality observed in this cohort [25]. Characteristics of this cohort are also consistent with previous studies that have identified risk factors for severe COVID-19 illness, including male sex and obesity, severe hypoxaemic respiratory failure requiring intensive respiratory support, and high rates of abnormal admission investigations, including lymphopenia, thrombocytopenia, and elevated C-reactive protein, D-dimer and ferritin [2, 26]. High rates of inpatient 
mMRC grade recovery to
pre-COVID-19 baseline

Adjusted OR

\begin{tabular}{cc}
$0.85(0.66-1.10)$ & 0.219 \\
$1.07(0.54-2.12)$ & 0.848 \\
$0.63(0.29-1.35)$ & 0.234 \\
$1.38(0.97-1.95)$ & 0.076 \\
$1.64(0.80-3.34)$ & 0.177 \\
$0.88(0.39-1.96)$ & 0.752 \\
$0.81(0.39-1.72)$ & 0.589 \\
$0.73(0.37-1.44)$ & 0.366 \\
$5.06(1.33-19.24)$ & $0.017 *$ \\
$1.03(0.82-1.28)$ & 0.823 \\
$1.04(0.84-1.29)$ & 0.706 \\
$0.86(0.39-1.92)$ & 0.715 \\
$0.96(0.77-1.19)$ & 0.677 \\
$1.22(0.51-2.94)$ & 0.657 \\
$1.36(0.56-3.30)$ & 0.503 \\
$0.94(0.46-1.92)$ & 0.872 \\
$2.26(0.95-5.37)$ & 0.066 \\
$1.51(1.13-2.02)$ & $0.00)^{*}$ \\
$2.04(0.55-7.62)$ & 0.290 \\
$0.92(0.23-3.70)$ & 0.909 \\
$8.04(3.62-17.84)$ & $0.000^{*}$ \\
$1.63(0.82-3.26)$ & 0.166 \\
$3.16(1.51-6.62)$ & $0.002^{*}$ \\
$2.60(1.31-5.19)$ & $0.007^{*}$ \\
$6.06(2.96-12.38)$ & $0.000^{*}$ \\
$1.28(0.57-3.32)$ & 0.554 \\
$1.36(0.63-3.11)$ & 0.432 \\
$0.90(0.43-2.96)$ & 0.781 \\
$1.55(0.73-1.88)$ & 0.256 \\
$7.03(2.77-2.89)$ & $0.000^{*}$ \\
$31.36(10.32-17.82)$ & $0.000^{*}$ \\
$9.71(3.23-95.36)$ & $0.000^{*}$ \\
$5.26(2.13-29.20)$ & $0.000^{*}$ \\
$2.21(1.57-12.97)$ & $0.000^{*}$ \\
& \\
\hline
\end{tabular}

Age

BAME

IMD

Obese (BMI >30 $\mathrm{kg} \cdot \mathrm{m}^{-2}$ )

Current smoker

Comorbid diabetes

Comorbid hypertension

Comorbid obstructive lung disease

Total comorbidities

Hospital LOS

$\mathrm{MO}_{2}$ maximum respiratory suppor

$\mathrm{PaO}_{2} / F_{\mathrm{IO}_{2}}$

ICU admission

ICU for IMV

No inpatient complications

Inpatient VTE

Follow-up RALE score

Normal CT

NRS breathlessness

NRS cough

NRS fatigue

NRS pain

NRS sleep disturbance

Positive 6-CIT

4MGS $<0.8 \mathrm{~m} \cdot \mathrm{s}^{-1}$

STS oxygen desaturation $\geqslant 4 \%$

Positive mental health screen

Positive PHQ-9

Positive GAD-7

Positive trauma screen

Positive PCFS

Pre-COVID mMRC

Current mMRC
Worst RALE score

STS repetitions $<2.5$ th percentile
PCFS grade $\geqslant 2$

Adjusted OR $(95 \% \mathrm{CI}) \quad$ p-valu

$1.12(0.56-2.22)$

$0.68(0.36-1.29)$

$1.16(0.81-1.68)$

$1.17(0.59-2.32)$

$1.15(0.54-2.43)$

$0.80(0.36-1.82)$

$0.81(0.38-1.71)$

$2.84(1.01-7.98)$

$1.09(0.86-1.38)$

$1.21(0.97-1.52)$

$0.48(0.22-1.03)$

$0.89(0.68-1.16)$

$2.57(1.12-5.90)$

$3.27(1.36-7.84)$

$0.82(0.40-1.69)$

$2.21(1.04-4.72)$

$1.09(0.81-1.48)$

$1.42(0.54-3.74)$

$0.62(0.16-2.47)$

$4.21(1.94-9.10)$

$1.79(0.91-3.51)$

$4.66(2.08-10.44)$

$6.54(2.98-14.35)$

$6.47(2.92-14.36)$

$0.96(0.32-2.89)$

$2.33(1.04-5.21)$

$0.83(0.42-1.65)$

$4.03(1.90-8.55)$

$12.13(5.03-29.26)$

$21.26(8.29-54.49)$

$13.20(5.03-34.65)$

7.41 (3.27-16.79)

$1.57(1.04-2.36)$

$2.48(1.74-3.54)$
Physiological functional

impairment

$\begin{array}{lllll}0.237 & 1.00(0.81-1.23) & 0.982 & 0.66(0.50-0.87) & 0.003^{*} \\ 0.748 & 0.84(0.39-1.81) & 0.656 & 0.68(0.30-1.54) & 0.358 \\ 0.243 & 1.81(0.95-3.46) & 0.071 & 0.77(0.32-1.82) & 0.548 \\ 0.417 & 0.73(0.52-1.03) & 0.072 & 1.53(0.98-2.40) & 0.061 \\ 0.645 & 0.75(0.37-1.50) & 0.415 & 1.61(0.66-3.89) & 0.292 \\ 0.715 & 1.27(0.59-2.74) & 0.546 & 1.46(0.57-3.72) & 0.431 \\ 0.601 & 1.58(0.73-3.39) & 0.243 & 1.33(0.52-3.37) & 0.550 \\ 0.576 & 1.62(0.69-3.84) & 0.271 & 1.76(0.73-4.25) & 0.210 \\ 0.047^{*} & 0.68(0.16-2.95) & 0.605 & 2.47(0.97-6.31) & 0.059 \\ 0.495 & 1.20(0.94-1.53) & 0.142 & 1.34(0.99-1.81) & 0.060 \\ 0.099 & 1.14(0.94-1.37) & 0.180 & 0.94(0.75-1.18) & 0.596 \\ 0.060 & 0.44(0.21-0.89) & 0.023^{*} & 0.85(0.32-2.22) & 0.735 \\ 0.393 & 1.00(0.80-1.25) & 0.999 & 0.88(0.67-1.15) & 0.338 \\ 0.026^{*} & 2.44(1.10-5.42) & 0.029 * & 1.26(0.47-3.42) & 0.643 \\ 0.008^{*} & 2.65(1.19-5.91) & 0.017 * & 1.23(0.45-3.36) & 0.688 \\ 0.592 & 0.74(0.37-1.52) & 0.416 & 1.12(0.45-2.80) & 0.808 \\ 0.040^{*} & 1.51(0.74-3.08) & 0.261 & 1.34(0.53-3.39) & 0.542 \\ 0.566 & 0.95(0.69-1.31) & 0.767 & 0.94(0.66-1.32) & 0.701 \\ 0.479 & 2.22(0.73-6.72) & 0.159 & 0.87(0.24-3.13) & 0.834 \\ 0.502 & 0.76(0.23-2.51) & 0.654 & 0.22(0.05-1.01) & 0.052 \\ 0.000^{*} & 1.77(0.82-3.83) & 0.149 & 4.34(1.58-11.95) & 0.004^{*} \\ 0.091 & 1.18(0.57-2.46) & 0.652 & 1.38(0.61-3.15) & 0.442 \\ 0.000^{*} & 1.09(0.51-2.33) & 0.827 & 3.58(1.32-9.70) & 0.012^{*} \\ 0.000^{*} & 0.77(0.38-1.59) & 0.487 & 9.62(3.65-25.38) & 0.000^{*} \\ 0.000^{*} & 1.32(0.66-2.65) & 0.437 & 7.24(2.42-21.62) & 0.000^{*} \\ 0.949 & 1.05(0.44-2.47) & 0.918 & 0.71(0.22-2.29) & 0.563 \\ 0.040^{*} & & & 3.86(1.52-9.77) & 0.004^{*} \\ 0.600 & & & 0.60(0.25-1.43) & 0.250 \\ 0.000^{*} & & 2.91(1.06-7.99) & 0.038^{*} \\ 0.000^{*} & 2.24(1.01-4.93) & 0.046^{*} & & \\ 0.000^{*} & 2.93(1.09-7.86) & 0.033^{*} & & \\ 0.000^{*} & 1.72(0.67-4.39) & 0.256 & & \\ 0.000^{*} & 1.84(0.80-4.27) & 0.153 & & \\ & 1.51(1.15-1.98) & 0.003^{*} & 2.89(2.09-4.01) & 0.000^{*} \\ 0.030^{*} & 1.49(1.06-2.08) & 0.020^{*} & 1.60(1.06-2.42) & 0.027^{*} \\ 0.000^{*} & 1.32(0.97-1.80) & 0.079 & 2.68(1.83-3.91) & 0.000^{*}\end{array}$

mMRC: modified Medical Research Council Dyspnoea (breathlessness) scale; PCFS: Post-COVID-19 Functional Status; BAME: Black, Asian or minority ethnic; IMD: index of multiple deprivation; $\mathrm{BMI}$ : body mass index; LOS: length of stay; $\mathrm{FMO}_{2}$ : facemask oxygen; $P_{\mathrm{aO}_{2}}$ : arterial oxygen tension; $F_{\mathrm{IO}_{2}}$ : inspiratory oxygen fraction; ICU: intensive care unit; IMV: invasive mechanical ventilation; VTE: venous thromboembolism; RALE: Radiographic Assessment of Lung Oedema; CT: computed tomography; NRS: numerical rating score; 6-CIT: six-item Cognitive Impairment Test; 4MGS: 4-m gait speed; STS: 1-min Sit-to-Stand test; PHQ-9: nine-item Patient Health Questionnaire; GAD-7: seven-item Generalised Anxiety Disorder assessment. \#: adjusted for age, sex and ethnicity. *: $p<0.05$ 


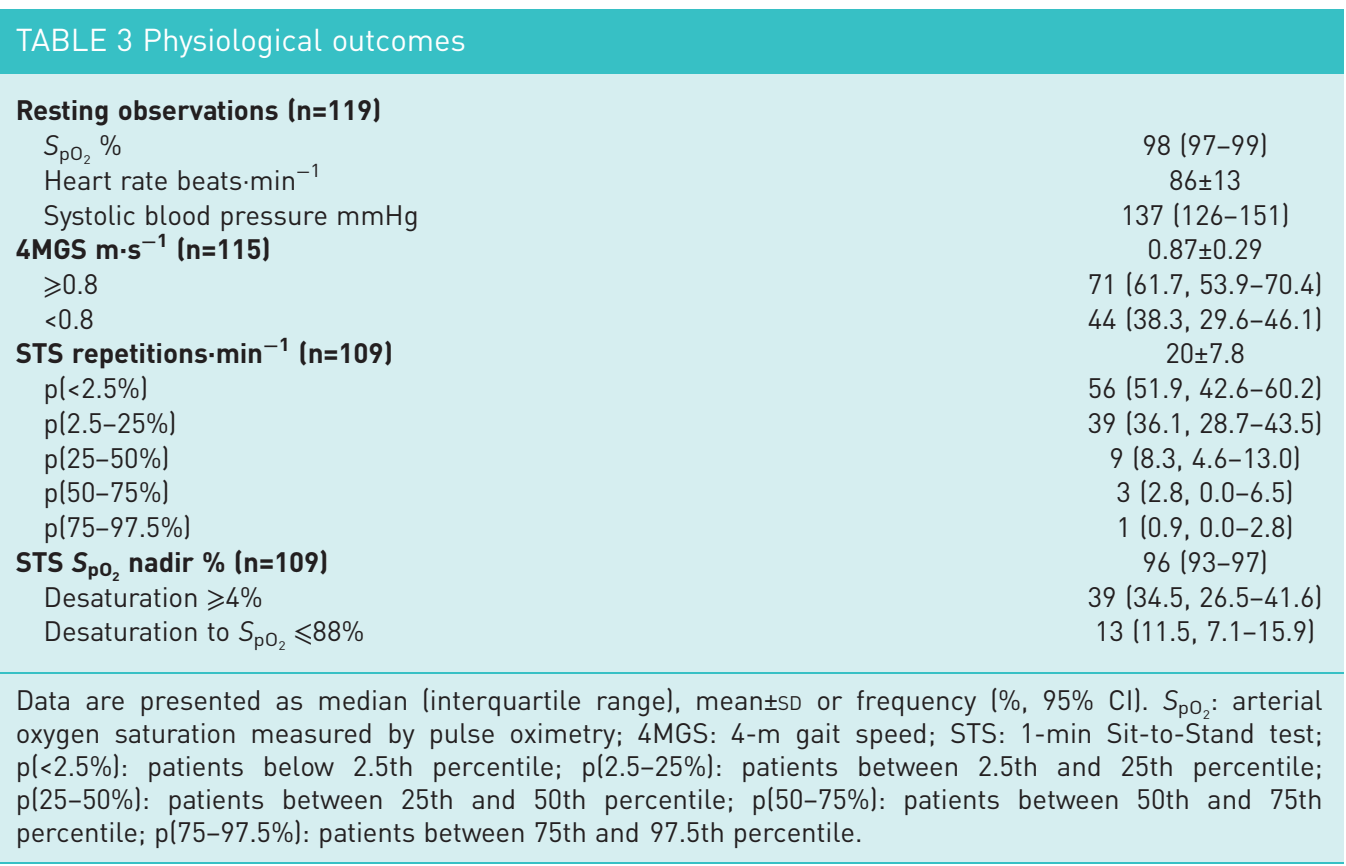

complications attributable to COVID-19 were observed, comparable to published data, including venous thromboembolism, acute kidney injury, deranged liver function and delirium [2, 27, 28]. Follow-up patients were younger with fewer comorbidities than the total cohort surviving to discharge, likely representing the characteristics of COVID-19 survivors, since these have been identified as risk factors for inpatient mortality in retrospective analyses $[29,30]$.

Few data are available on medium- and long-term effects of COVID-19 following hospital discharge. Our population characteristics are consistent with an Italian post-COVID-19 clinic cohort who had comparable quality-of-life impairment and persistent symptoms, although these patients were seen sooner following hospital discharge (mean \pm SD 36 \pm 13 days compared with median (IQR) 61 (51-67) days) [31]. Compared with studies evaluating radiological sequelae of MERS, the proportion of patients with chest radiograph resolution was much larger in our cohort (87\% measured at median (IQR) 76 (71-83) days from admission compared with $64 \%$ measured at 32-230 days) [32]. Data on post-COVID-19 CT findings are currently limited to within 3 weeks of symptom onset and indicate rapid evolution from unilateral multifocal ground-glass opacities to bilateral diffuse involvement to early reductions in ground-glass opacities by week 2 [33]. This is the first study to report CT outcomes post-discharge. We did not perform CT scans indiscriminately and would therefore anticipate that this selection would lead to

FIGURE 4 Box plots of Radiographic Assessment of Lung Oedema (RALE) scores at admission, worst during hospitalisation and follow-up. Observed values are indicated by circles. Shaded boxes indicate interquartile range with median indicated by the midline and mean indicated by a diamond. The lower/ upper whiskers represent the range to the minimum/maximum value or 1.5 times the median to the lower/ upper quartile, whichever is highest.

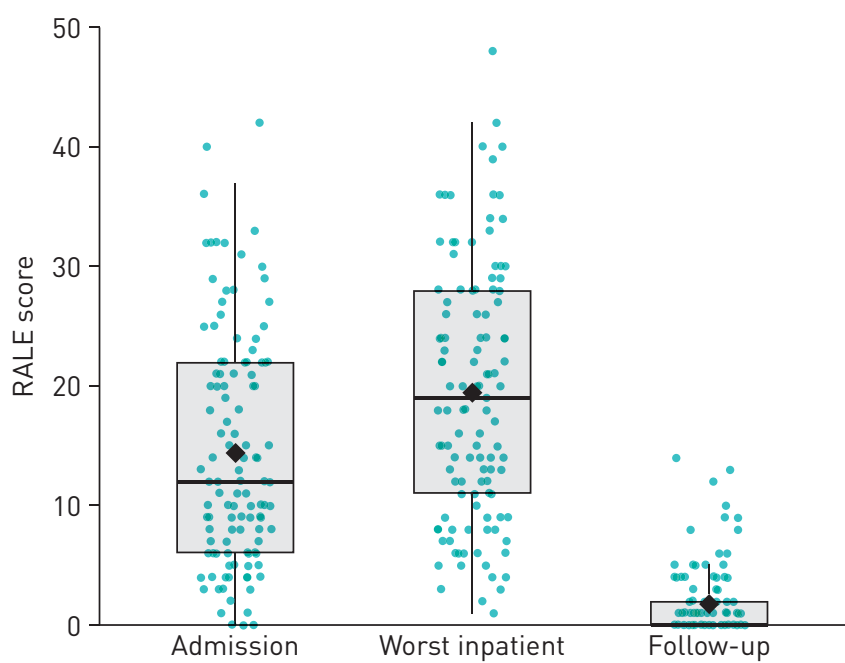


overrepresentation of interstitial abnormalities. Conversely, interstitial changes were less common in our cohort compared with patients with previous SARS, in whom 15 out of 24 (62\%) patients exhibited fibrotic changes on CT [5]. 15-year longitudinal data from SARS patients demonstrate resolution or stability of ground-glass changes with corresponding stability of total lung capacity and carbon monoxide diffusing capacity on lung function testing [34]. The long-term clinical implications and prognosis of COVID-19-related interstitial changes identified in the post-acute phase of illness remain unknown and labelling these abnormalities as lung fibrosis appears premature, particularly given the lack of association between presence of CT abnormalities and any measure of patient-reported or physiological functional impairment in this cohort.

Prevalence of persistent and burdensome physical symptoms, patient-reported and physiological functional impairment, and psychological sequelae was high in our cohort, and critical care admission and need for IMV were both associated with patient-reported and objective functional impairment. This is consistent with medium- and long-term data from ARDS and SARS survivors, in whom impaired quality of life and functional disability (measured using the 36-item Short Form Survey and 6-min walk test (6MWT)) were present at 3, 6 and 12 months following index hospitalisation [4, 7]. The burdens of patient-reported and physiological outcomes were high despite radiographic resolution occurring in the majority. Reasons for this remain speculative and may relate to our early assessment of patients with severe disease at median (IQR) 61 (51-67) days post-discharge. Long-term data on post-COVID-19 symptoms and functional outcomes are awaited. Clinically significant depression and anxiety were present in $18 \%$ and $22 \%$ of our cohort, respectively, and 25\% screened positive for PTSD, consistent with published data (31\% depression, $42 \%$ anxiety and $28 \%$ PTSD at 1 month post-discharge) [35].

\section{Implications for clinical practice}

Persistent symptoms of fatigue and sleep disturbance following severe COVID-19 pneumonia have implications for productivity, physical activity and mental health, reflected in the high rates of positive screening tests for anxiety, depression and PTSD observed in this cohort. While causative mechanisms for adverse mental health outcomes following COVID-19 infection have not been established, mental distress at the population level has increased in excess of anticipated trajectories during the COVID-19 pandemic [9]. ICU-acquired weakness is a major contributor to adverse long-term physical and psychological sequelae of critical illness [6] and likely applicable to COVID-19-survivors, particularly since conventional methods of rehabilitation and exercise have been limited [36]. It has also been speculated that biological pathophysiological mechanisms, relating to cerebral vascular inflammation and thrombosis, survivor guilt and isolation in COVID-19 survivors may also contribute to adverse mental health outcomes in this cohort [28, 35].

Chest radiography 12 weeks post-discharge is advocated by current guidelines to evaluate recovery, with face-to-face clinical assessment recommended only in those with abnormal radiographs or those who experienced severe illness [11]. However, the majority (87\%) of radiographs performed at follow-up demonstrated recovery (RALE score $<5$ ) despite the high prevalence of adverse patient-reported and/or physiological outcomes. The clinical implications of post-COVID-19 interstitial changes identified on CT remain unclear. Of note, parenchymal abnormalities in SARS survivors demonstrated recovery and stability in the 2 years following infection [34]. We strongly encourage integrated holistic assessment of COVID-19 survivors using both radiological and clinical reviews, which may be conducted face-to-face or virtually depending on healthcare resources and patient preference.

The battery of outcome measures implemented in this study is impractical for routine clinical use. Focused patient interview is an appropriate substitute for questionnaires, utilising the data presented, and we recommend routine application of functional exercise testing. The 4MGS and 1-min STS tests are reliable and validated methods of assessing exercise performance, are predictive of health-related quality of life, and correlate with other tests of functional capacity, including the incremental shuttle walk test (ISWT) and $6 \mathrm{MWT}[21,37,38]$. These self-paced tests were quick to perform, required simple instructions to the patients, minimal space, equipment and training, and were completed in the majority of cases with no adverse events. Importantly, they provided valuable clinical information regarding oxygen desaturation and exercise limitation that was not obtained from other sources, thus identifying a cohort of patients requiring CT evaluation and who may benefit from pulmonary rehabilitation or advice on graded and paced return to usual activities, although current facilities and evidence in this context remains unestablished [39, 40].

\section{Strengths and limitations}

We rapidly designed a pragmatic clinical service to prospectively collect comprehensive data on physical and psychological recovery from severe COVID-19 at a time when sequelae of the acute illness were unknown and healthcare resources were severely strained. We conducted systematic face-to-face 
assessments which enabled collection of a wealth of clinical, radiological, patient-reported and physiological data in a short period, with all outcome measures contributing to clinical decision making.

This study has some limitations. First, it was not possible to perform lung function testing in serial patients due to decontamination procedures required following this aerosol-generating procedure, limiting conclusions that can be drawn regarding respiratory sequelae of the disease [41]. Second, conventional field walking tests to evaluate exercise capacity (6MWT and ISWT) were impractical in the clinic setting. However, the 4MGS and STS tests are reliable, validated and pragmatic methods of assessing exercise performance that correlate with the 6MWT and ISWT, breathlessness, and health-related quality of life $[21,37,38]$. Third, given the ambiguity in current guidance we devised our own definition of "severe" COVID-19 pneumonia in order to assess patients at highest risk of complications and to rationalise resources, given the large number of COVID-19 admissions. This approach may have missed some patients with persistent symptoms or functional disability; however, those recovering from mild to moderate disease are now being invited to attend the post-COVID-19 clinic and we plan to report their outcomes in due course. Finally, these data were collected from a single, urban teaching centre which limits the generalisability of our results. However, this dataset is sufficiently large to provide reasonable estimates of post-COVID-19 sequelae while results of multicentre studies are awaited.

\section{Conclusions}

We have demonstrated that the burden of persistent symptoms, functional limitation and adverse mental health outcomes 2 months after severe COVID-19 pneumonia is high. Physiological impairments frequently persist despite apparent resolution of infiltrates on follow-up chest radiography. Routinely offering face-to-face post-COVID-19 follow-up assessment permitted inclusion of self-paced "bedside" physiological tests, which provided valuable clinical information on functional disability warranting further investigation that could not have been obtained from questionnaires or telephone consultations. With confirmed cases of COVID-19 continuing to rise worldwide, we recommend prompt face-to-face or virtual clinical assessment of COVID-19 pneumonia survivors to facilitate early recognition and management of physical and psychological sequelae in this vulnerable cohort.

Acknowledgements: We wish to acknowledge the dedication and commitment of the staff at King's College Hospital (London, UK) in rapidly developing a holistic face-to-face assessment pathway to care for survivors of COVID-19 at a time when the short-, medium- and long-term effects of the disease remained unknown. We also wish to acknowledge the specialist physiologists at King's College Hospital Chest Clinic, Tracey Fleming, Sheron Mathew, Alice Byrne and Mutahhara Choudhury, who embraced the incorporation of functional assessments in clinic to aid our understanding of COVID-19 recovery. We would also like to express our gratitude to the patients involved in this research, without whom our understanding of the medium-term implications of severe COVID-19 pneumonia would remain limited.

Data availability: Individual participant data that underlie the results reported in this article will be made available following de-identification upon reasonable request to the corresponding author beginning 3 months and ending 5 years following article publication, to researchers who provide a methodologically sound proposal (upon approval of an internal commission) to achieve aims in the approved proposal for scientific purposes following the signing of a data sharing agreement.

Author contributions: C.J. Jolley, M.D. Waller and F. Perrin conceived and designed the study. R.F. D'Cruz, F. Perrin, M.D. Waller, J. Periselneris, S. Norton, L-J. Smith, R. Madula, T. Patrick, D. Walder, W. McNulty, P. Macedo, K. Lee, A. Heitmann, R. Lyall, G. Warwick, J.B. Galloway, S.S. Birring, A. Patel, I. Patel and C.J. Jolley acquired, analysed or interpreted the data. R.F. D'Cruz, J. Periselneris, S. Norton and C.J. Jolley drafted the manuscript. R.F. D'Cruz, F. Perrin, M.D. Waller, J. Periselneris, S. Norton, L-J. Smith, R. Madula, T. Patrick, D. Walder, W. McNulty, P. Macedo, K. Lee, A. Heitmann, R. Lyall, G. Warwick, J.B. Galloway, S.S. Birring, A. Patel, I. Patel and C.J. Jolley critically revised the manuscript for important intellectual content. R.F. D'Cruz, S. Norton and C.J. Jolley performed statistical analysis. C.J. Jolley, J. Periselneris and S.S. Birring supervised the study. C.J. Jolley and R.F. D'Cruz are the guarantors of the study. The corresponding author attests that all listed authors meet authorship criteria and that no others meeting the criteria have been omitted.

Conflict of interest: R.F. D'Cruz has nothing to disclose. M.D. Waller has nothing to disclose. F. Perrin has nothing to disclose. J. Periselneris reports lecture fees from Gilead outside the submitted work. S. Norton has nothing to disclose. L-J. Smith has nothing to disclose. T. Patrick has nothing to disclose. D. Walder has nothing to disclose. A. Heitmann has nothing to disclose. K. Lee has nothing to disclose. R. Madula has nothing to disclose. W. McNulty has nothing to disclose. P. Macedo has nothing to disclose. R. Lyall has nothing to disclose. G. Warwick has nothing to disclose J.B. Galloway has nothing to disclose. S.S. Birring has nothing to disclose. A. Patel has nothing to disclose. I. Patel has nothing to disclose. C.J. Jolley has nothing to disclose.

Support statement: This study received no specific funding or grant from any agency in the public, commercial or not-for-profit sectors. R.F. D'Cruz is funded by a National Institute for Health Research Doctoral Research Fellowship. Funding information for this article has been deposited with the Crossref Funder Registry. 


\section{References}

1 World Health Organization. Timeline of WHO's response to COVID-19. 2020. www.who.int/news-room/detail/ 29-06-2020-covidtimeline Date last accessed: July 10, 2020.

2 Yang $\mathrm{X}, \mathrm{Yu} \mathrm{Y,} \mathrm{Xu} \mathrm{J,} \mathrm{et} \mathrm{al.} \mathrm{Clinical} \mathrm{course} \mathrm{and} \mathrm{outcomes} \mathrm{of} \mathrm{critically} \mathrm{ill} \mathrm{patients} \mathrm{with} \mathrm{SARS-CoV-2} \mathrm{pneumonia} \mathrm{in}$ Wuhan, China: a single-centered, retrospective, observational study. Lancet Respir Med 2020; 8: 475-481.

3 Mahase E. Covid-19: what do we know about "long covid"? BMJ 2020; 370: m2815.

4 Hui DS, Joynt GM, Wong KT, et al. Impact of severe acute respiratory syndrome (SARS) on pulmonary function, functional capacity and quality of life in a cohort of survivors. Thorax 2005; 60: 401-409.

5 Antonio GE, Wong KT, Hui DS, et al. Thin-section CT in patients with severe acute respiratory syndrome following hospital discharge: preliminary experience. Radiology 2003; 228: 810-815.

6 Herridge MS, Tansey CM, Matté A, et al. Functional disability 5 years after acute respiratory distress syndrome. N Engl J Med 2011; 364: 1293-1304.

7 Herridge MS, Cheung AM, Tansey CM, et al. One-year outcomes in survivors of the acute respiratory distress syndrome. N Engl J Med 2003; 348: 683-693.

8 Cuthbertson BH, Hull A, Strachan M, et al. Post-traumatic stress disorder after critical illness requiring general intensive care. Intensive Care Med 2004; 30: 450-455.

9 Pierce M, Hope H, Ford T, et al. Mental health before and during the COVID-19 pandemic: a longitudinal probability sample survey of the UK population. Lancet Psychiatry 2020; 7: 883-892.

10 Johns Hopkins University and Medicine Coronavirus Resource Centre. COVID-19 dashboard by the Center for Systems Science and Engineering (CSSE) at Johns Hopkins University (JHU). 2020. www.arcgis.com/apps/ opsdashboard/index.html\#/bda7594740fd40299423467b48e9ecf6 Date last accessed: August 7, 2020.

11 British Thoracic Society. British Thoracic Society guidance on respiratory follow up of patients with a clinico-radiological diagnosis of COVID-19 pneumonia. 2020. www.brit-thoracic.org.uk/document-library/qualityimprovement/covid-19/resp-follow-up-guidance-post-covid-pneumonia Date last accessed: July 6, 2020.

12 NHS Health Research Authority. Defining research: do I need NHS REC review? 2020. www.hra-decisiontools.org. uk/ethics Date last accessed: July 13, 2020.

13 Stevens JP, Baker K, Howell MD, et al. Prevalence and predictive value of dyspnea ratings in hospitalized patients: pilot studies. PLoS One 2016; 11: e0152601.

14 Boulet LP, Coeytaux RR, McCrory DC, et al. Tools for assessing outcomes in studies of chronic cough: CHEST guideline and expert panel report. Chest 2015; 147: 804-814.

15 Medical Research Council. Medical Research Council Dyspnoea scale. 1986. https://mrc.ukri.org/research/facilities-andresources-for-researchers/mrc-scales/mrc-dyspnoea-scale-mrc-breathlessness-scale Date last accessed: June 5, 2020.

16 Klok FA, Boon GJAM, Barco S, et al. The Post-COVID-19 Functional Status scale: a tool to measure functional status over time after COVID-19. Eur Respir J 2020; 56: 2001494.

17 Kroenke K, Spitzer RL, Williams JB. The PHQ-9: validity of a brief depression severity measure. J Gen Intern Med 2001; 16: 606-613.

18 Spitzer RL, Kroenke K, Williams JB, et al. A brief measure for assessing generalized anxiety disorder: the GAD-7. Arch Intern Med 2006; 166: 1092-1097.

19 Brewin CR, Rose S, Andrews B, et al. Brief screening instrument for post-traumatic stress disorder. Br J Psychiatry 2002; 181: 158-162.

20 Katzman R, Brown T, Fuld P, et al. Validation of a short Orientation-Memory-Concentration Test of cognitive impairment. Am J Psychiatry 1983; 140: 734-739.

21 Kon SS, Patel MS, Canavan JL, et al. Reliability and validity of 4-metre gait speed in COPD. Eur Respir J 2013; 42 333-340.

22 Strassmann A, Steurer-Stey C, Lana KD, et al. Population-based reference values for the 1-min sit-to-stand test. Int J Public Health 2013; 58: 949-953.

23 Warren MA, Zhao Z, Koyama T, et al. Severity scoring of lung oedema on the chest radiograph is associated with clinical outcomes in ARDS. Thorax 2018; 73: 840-846.

24 Intensive Care National Audit and Research Centre. ICNARC Report on COVID-19 in Critical Care; England Wales and Northern Ireland, 25 September 2020. 2020. www.icnarc.org/Our-Audit/Audits/Cmp/Reports Date last accessed: September 28, 2020.

25 Docherty AB, Harrison EM, Green CA, et al. Features of 20133 UK patients in hospital with covid-19 using the ISARIC WHO Clinical Characterisation Protocol: prospective observational cohort study. BMJ 2020; 369: m1985.

26 Galloway JB, Norton S, Barker RD, et al. A clinical risk score to identify patients with COVID-19 at high risk of critical care admission or death: an observational cohort study. J Infect 2020; 81: 282-288.

27 Bilaloglu S, Aphinyanaphongs Y, Jones S, et al. Thrombosis in hospitalized patients with COVID-19 in a New York City health system. JAMA 2020; 324: 799-801.

28 Varatharaj A, Thomas N, Ellul MA, et al. Neurological and neuropsychiatric complications of COVID-19 in 153 patients: a UK-wide surveillance study. Lancet Psychiatry 2020; 7: 875-882.

29 Grasselli G, Zangrillo A, Zanella A, et al. Baseline characteristics and outcomes of 1591 patients infected with SARS-CoV-2 admitted to ICUs of the Lombardy Region, Italy. JAMA 2020; 323: 1574-1581.

30 Guan WJ, Liang WH, Zhao Y, et al. Comorbidity and its impact on 1590 patients with COVID-19 in China: a nationwide analysis. Eur Respir J 2020; 55: 2000547.

31 Carfi A, Bernabei R, Landi F, et al. Persistent symptoms in patients after acute COVID-19. JAMA 2020; 324: 603-605.

32 Das KM, Lee EY, Singh R, et al. Follow-up chest radiographic findings in patients with MERS-CoV after recovery. Indian J Radiol Imaging 2017; 27: 342-349.

33 Shi H, Han X, Jiang N, et al. Radiological findings from 81 patients with COVID-19 pneumonia in Wuhan, China: a descriptive study. Lancet Infect Dis 2020; 20: 425-434.

34 Zhang $\mathrm{P}, \mathrm{Li} \mathrm{J}$, Liu $\mathrm{H}$, et al. Long-term bone and lung consequences associated with hospital-acquired severe acute respiratory syndrome: a 15-year follow-up from a prospective cohort study. Bone Res 2020; 8: 8 .

35 Gennaro Mazza M, De Lorenzo R, Conte C, et al. Anxiety and depression in COVID-19 survivors: role of inflammatory and clinical predictors. Brain Behav Immun 2020; 89: 594-600.

36 Thornton J. Covid-19: the challenge of patient rehabilitation after intensive care. BMJ 2020; 369: m1787. 
37 Ozalevli S, Ozden A, Itil O, et al. Comparison of the Sit-to-Stand Test with 6 min walk test in patients with chronic obstructive pulmonary disease. Respir Med 2007; 101: 286-293.

38 Puhan MA, Siebeling L, Zoller M, et al. Simple functional performance tests and mortality in COPD. Eur Respir J 2013; 42: 956-963.

39 Polastri M, Nava S, Clini E, et al. COVID-19 and pulmonary rehabilitation: preparing for phase three. Eur Respir J 2020; 55: 2001822

40 White PD, Goldsmith KA, Johnson AL, et al. Comparison of adaptive pacing therapy, cognitive behaviour therapy, graded exercise therapy, and specialist medical care for chronic fatigue syndrome (PACE): a randomised trial. Lancet 2011; 377: 823-836.

41 European Respiratory Society. Recommendation from ERS Group 9.1 (Respiratory function technologists/ Scientists). Lung function testing during COVID-19 pandemic and beyond. 2020. https://ers.app.box.com/s/ zs1uu88wy51monr0ewd990itoz4tsn2h Date last accessed: July 8, 2020. 